\title{
Fixed high dose right unilateral electroconvulsive therapy produced a higher antidepressant response rate than titrated dosing
}

\author{
McCall WVReboussin DM, Weiner RD, et al. Titrated moderately suprathreshold vs fixed high-dose right unilateral \\ electroconvulsive therapy: acute antidepressant and cognitive effects. Arch Gen Psychiatry 2000 May;57:438-44.
}

QUESTION: In patients with a major depressive disorder (MDD), how do titrated moderately suprathreshold and fixed high dose right unilateral (RUL) electroconvulsive therapy (ECT) compare for acute antidepressant response and cognitive side effects?

\section{Design}

Randomised \{allocation concealed*\}†, blinded (\{treatment team, patients $\} \dagger$, and outcome assessors), ${ }^{*}$ controlled trial with 1-2 days of follow up after the last RUL ECT session.

\section{Setting}

Tertiary care centre in Winston-Salem, North Carolina, USA.

\section{Patients}

72 patients (90\% outpatients) who were $\geq 18$ years of

Source of funding: $U S$ National Institute of Mental Health.

For correspondence: Dr W V McCall,

Department of

Psychiatry, Wake Forest University School of

Medicine, Medical

Center Boulevard,

Winston-Salem, $N C$

27157, USA. Fax +1

3367166830 . age (mean age 64 y, $75 \%$ women) and met DSM-III-R criteria for MDD. Exclusion criteria were a history of functional psychosis, active substance abuse, mental retardation or neurological illness, ECT in the past 4 months, score of $<18$ on the Mini-Mental State Examination (MMSE), score of $<20$ on the Hamilton Rating Scale for Depression (HRSD), need for bilateral ECT because of extreme severity of illness, refusal to cooperate with testing, or predominantly left handedness. Follow up was complete for antidepressant response and $88 \%$ for cognitive side effects.

Titrated right unilateral electroconvulsive therapy (RUL ECT) v fixed high dose RUL ECT for major depression at 1-2 days:

\begin{tabular}{lllll} 
Outcomes & $\begin{array}{l}\text { Titrated } \\
\text { RUL ECT }\end{array}$ & $\begin{array}{l}\text { Fixed dose } \\
\text { RUL ECT }\end{array}$ & RBR (95\% CI) & NNH (Cl) \\
\hline Antidepressant response & $39 \%$ & $67 \%$ & $42 \%(9$ to 64$)$ & 4 (3 to 20) \\
\hline & & & RRR (CI) & NNT (CI) \\
\hline Cognitive disturbance & $6.7 \%$ & $30 \%$ & $78 \%(20$ to 94$)$ & 5 (3 to 22) \\
\hline
\end{tabular}

‡RBR=relative benefit reduction. Other abbreviations defined in glossary; RBR, RRR, NNH, NNT, and $\mathrm{Cl}$ calculated from data provided by author.

\section{Intervention}

RUL ECT seizure threshold (ST) was determined at the first session. Patients were then allocated to titrated RUL ECT at 2.25 times the initial ST (mean dose 136 millicoulombs [mC]) $(n=36)$ or a fixed high dose of 403 $\mathrm{mC}$ of RUL ECT $(\mathrm{n}=36)$. The attending physician determined the number of treatments.

\section{Main outcome measures}

Antidepressant response (decrease of $\geq 60 \%$ in HRSD score and a final score of $\leqslant 12$ ), and global cognitive disturbance (decrease of $\geq 5$ points on the MMSE) 1-2 days after the course of ECT, and retrograde and anterograde memory function.

\section{Main results}

Analysis of the antidepressant response was by intention to treat. The rate of antidepressant response was greater in the fixed high dose RUL ECT group than in the titrated group $(\mathrm{p}=0.02)$, but the high dose group had more cognitive disturbance $(\mathrm{p}=0.02)$ (table). Autobiographical memory was greater in the titrated RUL ECT group than in the fixed high dose group (percentage of autobiographical recall $66.1 \%$ v $53.5 \%, \mathrm{p}=0.01)$.

\section{Conclusion}

In patients with a major depressive episode, titrated moderately suprathreshold right unilateral (RUL) electroconvulsive therapy (ECT) produced a lower antidepressant response rate, but fewer cognitive side effects than fixed high dose RUL ECT.

*See glossary.

$\dagger$ Information provided by author.

\section{COMMENTARY}

The studies by Sackeim et al and McCall et al address the continuing controversy about the benefits and risks of UL ECT and BL ECT. The report by Sackeim et al is the third since 1987 by these authors seeking to increase the efficacy of UL ECT and to sustain its immediate lesser cognitive effects compared with bilateral placement (BL ECT). RUL ECT with dosages 5 times ST is reported to be equal in efficacy to BL ECT at 2.5 times ST, and with fewer short term cognitive side effects. The sample size (20 patients per group), however, is too small to ensure that clinically important differences do not exist. Cognition was assessed immediately after seizure and 2-7 days after the last treatment, and favoured RUL ECT over BL ECT. For most patients, however, the immediate cognitive effects diminish after 2 months and this benefit may not be sufficient to justify RUL ECT given the uncertainties about its efficacy compared with BL ECT.

The stimulus dosing of BL ECT was applied at 2.5 times ST in the study by Sackeim $e t$ al, but BL ECT has been shown to be effective at 1.5 times ST in a previous study by these authors. ${ }^{1}$ The selection of a higher BL ECT dosing intensity ensured efficacy, but also greater cognitive side effects; RUL ECT at a high dosage stimulus may confer a cognitive advantage in this regard. Clinical application of these results is problematic because the BL ECT dosage in this study markedly exceeds that used in most treatment settings.

Even if efficacy is equivalent, and cognitive side effects are lessened, such high dosing will be impractical for many clinicians. For example, the US Food and Drug Administration regulations on ECT output would not permit the highest RUL ECT intensities used in this study for about $20 \%$ of patients. 